\title{
Effect of oral phytoestrogen on androgenicity and insulin sensitivity in postmenopausal women
}

\author{
C. C. Lee ${ }^{1,2}$, C. J. Bloem³ ${ }^{3}$ J. Z. Kasa-Vubu \& L.-J. Liang ${ }^{1}$ \\ ${ }^{1}$ Department of Medicine, David Geffen School of Medicine, UCLA, Los Angeles, CA, USA \\ 2 VA Greater Los Angeles Healthcare System, Geriatric Research Education and Clinical Center, Los Angeles, CA, USA \\ ${ }^{3}$ Department of Cardiac Surgery, University of Michigan, Ann Arbor, MI, USA \\ ${ }^{4}$ Division of Pediatric Endocrinology, Department of Pediatrics, University of Michigan Health System, Ann Arbor, MI, USA
}

Aim: The aim of this study was to determine and compare the effect of treatment with transdermal oestrogen and phytoestrogen on insulin sensitivity and sex hormone-binding globulin (SHBG) levels in healthy postmenopausal women.

Methods: Forty-three healthy postmenopausal women aged $68 \pm 7$ (mean \pm SD) years who were not receiving hormonal replacement therapy completed a 3 month randomized drug therapy study. The participants were randomized to one of four groups: $0.05 \mathrm{mg}$ or $0.1 \mathrm{mg}$ transdermal oestrogen/day, or 40 or $80 \mathrm{mg}$ oral phytoestrogen (Promensil)/day insulin sensitivity was indirectly measured using the quantitative insulin sensitivity check index (QUICKI). SHBG, total testosterone, oestradiol, and fasting glucose and insulin levels for calculation of insulin sensitivity were obtained at baseline and at monthly intervals during the 3 months of therapy.

Results: In healthy nondiabetic postmenopausal women, the rate of change in QUICKI was significantly different between the red clover based phytoestrogen and transdermal oestrogen groups, so that after three months of therapy, QUICKI with red clover based phytoestrogen therapy was lower than that in the transdermal oestrogen group, $p=0.01$. Red clover based phytoestrogen therapy was not associated with any changes in SHBG levels whereas transdermal estrogen therapy significantly increased SHBG levels, $p=0.05$.

Conclusions: In contrast to transdermal oestrogen therapy, oral phytoestrogen therapy does not decrease androgenicity and is associated with a decrease in insulin sensitivity. These effects are similar to those of raloxifene and consistent with phytoestrogen's selective oestrogen receptor modulator properties.

Keywords: androgenicity insulin sensitivity, phytoestrogen, postmenopausal women, transdermal oestrogen

Date submitted 10 March 2011; date of first decision 24 April 2011; date of final acceptance 26 September 2011

\section{Introduction}

Although evidence supports that oestrogen therapy improves insulin sensitivity, ameliorates arterial compliance and promotes a more favorable lipid profile in women, current evidence concerning the adverse consequences of hormonal replacement in postmenopausal women has renewed interest in alternatives to oestrogen therapy. These alternatives include herbal products containing compounds with oestrogenic properties derived from plants or phytoestrogens. One of the most widely distributed phytoestrogens currently sold is a red clover botanical supplement [1]. Much of the positive data regarding the effects of phytoestrogens on menopausal symptoms, cardiac risk factors and cancer prevention are derived from observational studies. Prospective studies to date suggest that red clover based phytoestrogens may have similar effects to oestrogen therapy to improve metabolic risk factors such as vascular endothelial function in postmenopausal women with type 2 diabetes, arterial compliance in healthy

Correspondence to: Cathy C. Lee, VA Greater Los Angeles Healthcare System, 11301 Wilshire Blvd., GRECC 11-G, Los Angeles, CA 90073, USA.

E-mail: cathy.c.lee@ucla.edu postmenopausal women and possibly the lipid profile. Previous prospective studies on the effects of red clover based phytoestrogens on insulin resistance, however, have not had consistent results and are more difficult to interpret because insulin resistance was not the primary outcome. These limited studies on insulin resistance were also performed in different study populations, postmenopausal women with type 2 diabetes [2] or premenopausal women [3], therefore difficult to compare.

The objective of this randomized study was to directly compare the effects of a red clover based oral phytoestrogen with transdermal oestrogen on insulin sensitivity in nondiabetic postmenopausal women. Secondarily, we wanted to assess the effects of these drugs on postmenopausal hormone levels and levels of sex hormone-binding globulin (SHBG).

\section{Methods}

\section{Subjects}

Forty-three healthy postmenopausal women who were not on hormone replacement therapy were enrolled. Subjects were recruited through the Human Subjects Core of the 
University of Michigan Geriatrics Center and newspaper advertisement. Subjects were screened before study entry with a medical history, physical examination and laboratory tests, which included a complete blood count, thyroid-stimulating hormone and routine chemistries. Subjects were excluded from participation if they had any history of deep venous thrombosis, pulmonary embolism, uterine cancer, breast cancer; or had contraindications to oestrogen; or had evidence from the history, physical examination, or laboratory testing of other significant underlying medical or psychiatric illness. No subjects had a fasting glucose level that would indicate a diagnosis of diabetes and no subjects reported polycystic ovarian disease in their medical history. Each subject gave written informed consent that was approved by the University of Michigan Institutional Review Board.

\section{Study Protocol}

Subjects who fulfilled eligibility criteria were randomized to one of four groups: (i) $0.05 \mathrm{mg}$ transdermal oestrogen/day, (ii) $0.1 \mathrm{mg}$ transdermal oestrogen/day, (iii) $40 \mathrm{mg}$ oral phytoestrogen (Promensil)/day or (iv) $80 \mathrm{mg}$ oral phytoestrogen/day. These doses of transdermal oestrogen were chosen because they are the standard doses used in clinical practice for hormone therapy in postmenopausal women. The doses of oral phytoestrogen were chosen based on the results of a previous meta-analysis demonstrating that a dose of $47 \mathrm{mg}$ /day of soy isoflavone had clinically significant effects on metabolic parameters related to cardiovascular health [4].

A 12-week study was proposed for two reasons. First, a decrease in serum lipids and improvement of insulin sensitivity has been shown after 12 weeks of therapy with transdermal oestrogen [5] and oral soy isoflavone [4]. Secondly, because combination therapy with oestrogen and progesterone would be complicated by the known confounding of progesterone to decrease insulin sensitivity [6], the exposure to unopposed oestrogen was limited.

All subjects underwent fasting glucose and insulin measurements at baseline and at monthly intervals for 3 months during drug therapy, as well as hormone measurements for SHBG, oestradiol, estrone, total and free testosterone.

\section{Measurement of Insulin Sensitivity}

Blood samples for glucose and insulin were collected in glass tubes containing sodium heparin, stored on ice and separated immediately after collection. Plasma was stored at $-70^{\circ} \mathrm{C}$ until assay. Plasma glucose was measured using the autoanalyser glucose oxidase method and plasma insulin by RIA in the Core Laboratory of the Michigan Diabetes Research and Training Center. Insulin sensitivity was determined using the quantitative insulin sensitivity check index (QUICKI) calculated by the equation: $1 /\{\log [$ fasting insulin $(\mu \mathrm{U} / \mathrm{ml})]$ $+\log [$ fasting glucose $(\mathrm{mg} / \mathrm{dl})]\}[7]$.

\section{Measurement of Androgenicity}

Plasma testosterone and oestradiol were measured using chemiluminescence in the Core Laboratory of the University of Michigan General Clinical Research Center. Plasma SHBG levels were measured using a solid-phase, twosite chemiluminescent enzyme immunometric assay for use with the Immulite Automated Analyzer (Immulite SHBG, Diagnostic Product Corp., Los Angeles, CA, USA). Plasma samples were stored at $-70^{\circ} \mathrm{C}$ until assayed. All samples were analysed in the same assay. The sensitivity of the SHBG assay was $0.2 \mathrm{nmol} / \mathrm{l}$. The intra-assay and interassay coefficients of variation were 6.5 and $8.7 \%$, respectively.

\section{Data and Statistical Analysis}

All subjects were analysed on an intent-to-treat basis. Demographic and baseline characteristics were summarized using descriptive statistics ( $\mathrm{N}$, mean and standard error) and compared among the four subject groups using analysis of variance (ANOVA). Body mass index (BMI, $\mathrm{kg} / \mathrm{m}^{2}$ ) was determined by dividing subjects' weight in kilograms by their height in metres squared. Percentage change of SHBG from baseline was calculated at each follow-up for each of the four subject groups, and the mean bars (with standard error) for low transdermal oestrogen versus high dose and for low dose oral phytoestrogen versus high dose are shown in figure 2. Values are presented as mean \pm standard error of the mean, except for the subject characteristics and body composition measurements, which are presented as mean \pm standard deviation.

Mixed-effects regression models were used to compare the effects of drug therapy on the following variables of interest: SHBG, oestradiol, testosterone, glucose, insulin and insulin sensitivity as measured by QUICKI. Covariates included in these models were age, BMI, drug, time and drug-by-time. The mixed-effect regression models properly accounted for correlations among repeated measures, and also allowed for heterogeneous variances. Data were analysed using SAS version 9.2 (SAS Institute Inc., Cary, NC, USA) and GraphPad Prism version 4.03 for Windows (GraphPad Software, San Diego, CA, USA).

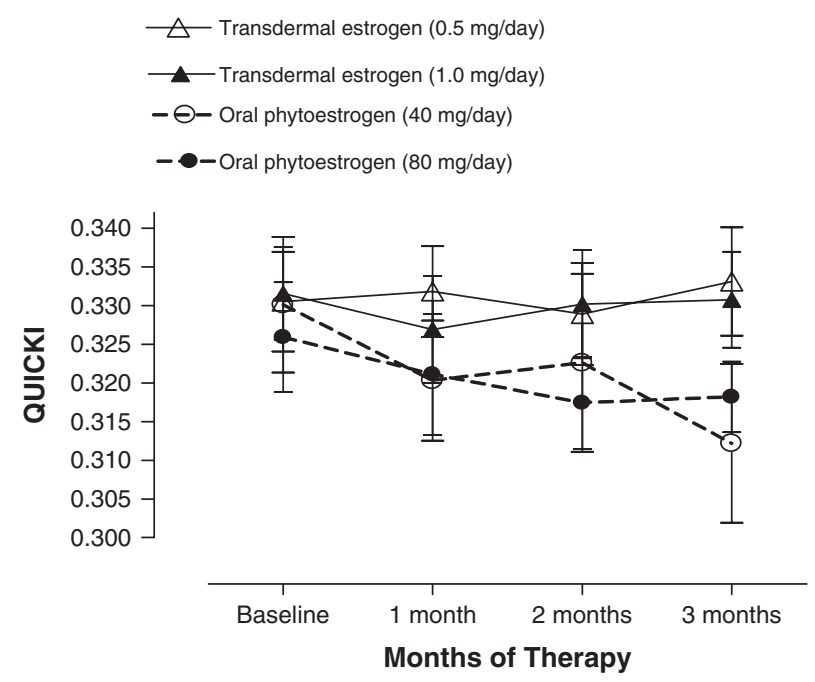

Figure 1. Change in insulin sensitivity as measured by quantitative insulin sensitivity check index (QUICKI) over 3 months of drug therapy with either transdermal oestradiol or oral phytoestrogen. 


\section{Results}

The mean age of the women studied was 68 years with a range between 60 and 83 years, and they were somewhat overweight as reflected by their mean BMI of $27 \mathrm{~kg} / \mathrm{m}^{2}$. There were no statistically significant differences with respect to age, BMI, or hormone status at baseline between the four subject groups (Table 1). In addition, there were no statistically significant differences with respect to fasting glucose, fasting insulin or QUICKI between the four subject groups at baseline (Table 1).

As shown in figure 1, the rate of change in QUICKI was significantly different between the red clover based phytoestrogen and transdermal estrogen groups, so that after 3 months of drug therapy, QUICKI with red clover based phytoestrogen therapy was lower than that in the transdermal estrogen group (adjusted $\mathrm{p}=0.01$ ). We found that age and BMI were significantly associated with SHBG levels (age $\mathrm{p}=0.03$, BMI $\mathrm{p}=0.001$ ), and BMI was significantly associated with QUICKI ( $\mathrm{p}<0.0001$ ) (Table 2). Also shown in Table 2, positive rates of change in QUICKI (increased insulin sensitivity) were found for both doses of transdermal oestrogen, whereas negative rates of change in QUICKI (decreased insulin sensitivity) were found for both doses of oral phytoestrogens. There was no statistically significant dose effect of either therapy.

After 3 months of drug therapy, fasting glucose levels were significantly higher in the oral phytoestrogen groups compared with the transdermal oestrogen groups (unadjusted drug effect, $\mathrm{p}=0.01$ ). However, after adjustment for age and BMI, the effects of transdermal oestrogen and oral phytoestrogen therapy on fasting glucose and insulin levels were no longer significant.

As expected, the transdermal oestrogen groups significantly increased SHBG levels (Table 2, figure 2, adjusted $\mathrm{p}=0.05$ ). Neither dose of oral phytoestrogen therapy changed SHBG levels or oestradiol levels. There was no significant change in total testosterone levels with either transdermal oestrogen or oral phytoestrogen.

\section{Discussion}

The present study showed that in contrast to transdermal oestrogen's effect to maintain insulin sensitivity, red clover based phytoestrogen therapy was associated with a significant decrease in insulin sensitivity in healthy nondiabetic postmenopausal women. We did not find any dose effect; therefore, the high and low doses of each therapy were combined for some of the analyses.

Postmenopausal oestrogen therapy is generally believed to improve insulin sensitivity and may decrease the risk for type 2 diabetes. Because red clover based phytoestrogen therapy shares some other positive effects of oestrogen therapy, we postulated that it would also improve insulin sensitivity. Soy protein, as well as genistein specifically, has been shown to decrease insulin resistance in postmenopausal women with and without type 2 diabetes $[8,9]$. Different red clover based phytoestrogens have been shown to have no effect on indirect measures of insulin sensitivity in postmenopausal women with type 2 diabetes and premenopausal women. No previous studies have directly examined the effect of red clover based phytoestrogens on insulin sensitivity in healthy postmenopausal women. In contrast to the results of the few limited prior studies on red clover based phytoestrogens, in the present study red clover based phytoestrogen therapy was shown to significantly reduce insulin sensitivity.

The effects of red clover based phytoestrogens on insulin sensitivity observed in our present study are similar to those previously reported for raloxifene [10], and therefore consistent with phytoestrogen's selective oestrogen receptor modulator (SERM) properties. Phytoestrogens have a similar molecular structure to other SERMs and to oestrogen, are known to bind to oestrogen receptors and compete with the oestradiol receptor complex for nuclear binding. This appears to account for the ability of phytoestrogens to act as either an oestrogen agonist or antagonist [11]. Phytoestrogens may also differ from oestradiol by being more potent oestrogen receptor beta agonists rather than oestrogen receptor alpha agonists [12]. In addition to the differential activity of phytoestrogens at the oestrogen receptor and competition with oestradiol at the cellular level, phytoestrogen absorption from the intestinal tract may stimulate production of SHBG thus lowering free oestradiol levels in the circulation. However, we did not see any effect of red clover based phytoestrogens on SHBG, oestradiol or testosterone levels in this study. Another potential mechanism for the differential activity of phytoestrogens may be because of alteration of aromatase activity leading to a decrease in active oestrogen concentrations. This may be because of structural

Table 1. Baseline subject characteristics.*

\begin{tabular}{|c|c|c|c|c|}
\hline & $\begin{array}{l}\text { Transdermal oestrogen } \\
(0.5 \mathrm{mg}), \mathrm{N}=10\end{array}$ & $\begin{array}{l}\text { Transdermal oestrogen } \\
(1.0 \mathrm{mg}), \mathrm{N}=11\end{array}$ & $\begin{array}{l}\text { Oral isoflavone } \\
(40 \mathrm{mg}), \mathrm{N}=10\end{array}$ & $\begin{array}{l}\text { Oral isoflavone } \\
(80 \mathrm{mg}), \mathrm{N}=12\end{array}$ \\
\hline Age (years) & $72 \pm 8$ & $65 \pm 5$ & $69 \pm 7$ & $67 \pm 6$ \\
\hline BMI (kg/m2) & $26 \pm 5$ & $29 \pm 6$ & $26 \pm 4$ & $26 \pm 5$ \\
\hline SHBG (nM) & $83.7 \pm 30$ & $55.0 \pm 26$ & $85.5 \pm 45$ & $56.2 \pm 24$ \\
\hline Total testosterone (nM) & $0.016 \pm 0.01$ & $0.013 \pm 0.01$ & $0.005 \pm 0.0008$ & $0.005 \pm 0.001$ \\
\hline Oestradiol (pM) & $135.5 \pm 79.0$ & $62.4 \pm 8.7$ & $44.8 \pm 5.4$ & $61.5 \pm 4.4$ \\
\hline Glucose (mM) & $5.3 \pm 0.2$ & $5.2 \pm 0.2$ & $5.1 \pm 0.2$ & $5.5 \pm 0.2$ \\
\hline Insulin (pM) & $85.84 \pm 12.0$ & $84.0 \pm 8.7$ & $100.8 \pm 22.5$ & $98.9 \pm 18.4$ \\
\hline QUICKI & $0.331 \pm 0.006$ & $0.332 \pm 0.006$ & $0.330 \pm 0.009$ & $0.326 \pm 0.007$ \\
\hline
\end{tabular}

BMI, body mass index; SHBG, sex hormone-binding globulin; QUICKI, quantitative insulin sensitivity check index.

* Group comparisons were carried out using ANOVA. No statistically significant differences among the four subject groups were found for all variables. 
Table 2. Results from mixed-effects models.

\begin{tabular}{|c|c|c|c|c|}
\hline \multirow[b]{3}{*}{ Parameter } & \multicolumn{4}{|l|}{ Outcome } \\
\hline & \multicolumn{2}{|l|}{ SHBG } & \multicolumn{2}{|l|}{ QUICKI } \\
\hline & Estimate (SE) & $\mathrm{p}$ & Estimate (SE) & $\mathrm{p}$ \\
\hline Age & $1.51(0.69)$ & 0.03 & $-0.12 \mathrm{E}-3(0.4 \mathrm{E}-3)$ & 0.7876 \\
\hline BMI & $-2.86(0.86)$ & 0.0013 & $-2.57 \mathrm{E}-3(0.5 \mathrm{E}-3)$ & $<0.0001$ \\
\hline Drug (baseline) & & 0.16 & & 0.51 \\
\hline Transdermal oestrogen $(0.5 \mathrm{mg})$ & $75.2(10.5)$ & & $0.33(0.006)$ & \\
\hline Transdermal oestrogen (1.0 mg) & $68.6(9.96)$ & & $0.34(0.006)$ & \\
\hline Oral phytoestrogen $(40 \mathrm{mg})$ & $79.8(9.47)$ & & $0.33(0.007)$ & \\
\hline Oral phytoestrogen ( $80 \mathrm{mg})$ & $52.9(8.38)$ & & $0.32(0.005)$ & \\
\hline Slope & & 0.06 & & 0.08 \\
\hline Transdermal oestrogen $(0.5 \mathrm{mg})$ & $3.50(2.99)$ & & $0.51 \mathrm{E}-3(0.001)$ & \\
\hline Transdermal oestrogen $(1.0 \mathrm{mg})$ & $7.22(3.10)$ & & $0.21 \mathrm{E}-3(0.001)$ & \\
\hline Oral phytoestrogen (40 mg) & $-2.41(2.45)$ & & $-5.08 \mathrm{E}-3(0.002)$ & \\
\hline Oral phytoestrogen $(80 \mathrm{mg})$ & $2.67(2.09)$ & & $-2.64 \mathrm{E}-3(0.001)$ & \\
\hline \multicolumn{5}{|l|}{ Difference in slopes } \\
\hline Transdermal oestrogen: $\mathrm{HI}-\mathrm{LO}$ & $3.73(4.31)$ & 0.39 & $-0.29 \mathrm{E}-3(0.002)$ & 0.87 \\
\hline Oral phytoestrogen: $\mathrm{HI}$ - LO & $5.08(3.22)$ & 0.12 & $2.43 \mathrm{E}-3(0.003)$ & 0.38 \\
\hline Oestrogen-oral isoflavone & $5.23(2.69)$ & 0.05 & $4.22 \mathrm{E}-3(0.002)$ & 0.01 \\
\hline
\end{tabular}

BMI, body mass index; SHBG, sex hormone-binding globulin; QUICKI, quantitative insulin sensitivity check index.

Transdemal estrogen $(0.5 \mathrm{mg} /$ day $)$

[E] Transdermal estrogen (1.0 mg/day)

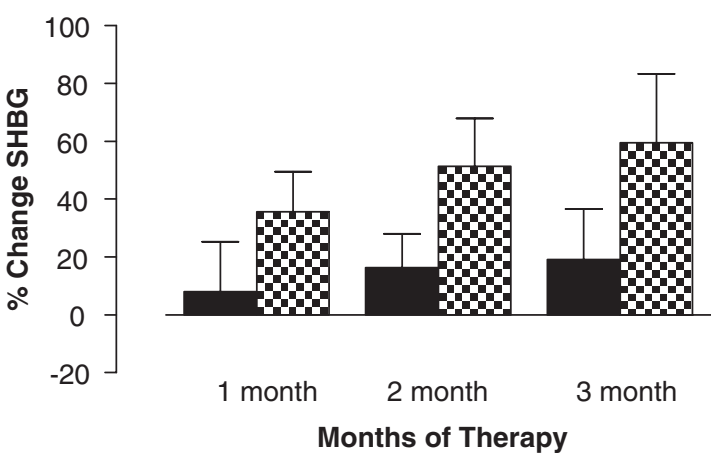

Oral phytoestrogen (40 mg/day)

Brin Oral phytoestrogen (80 mg/day)

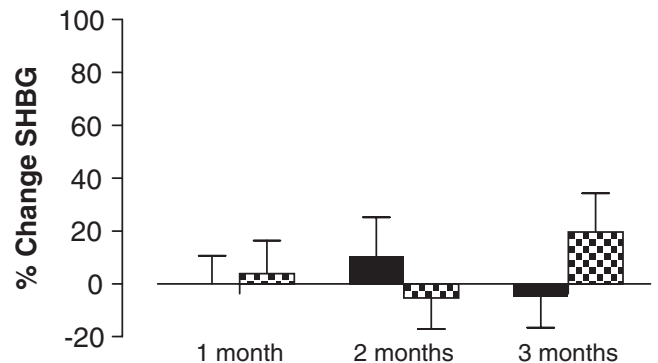

Months of Therapy

Figure 2. Percentage of change in sex hormone-binding globulin (SHBG) levels over 3 months of drug therapy with either transdermal oestradiol or oral phytoestrogen.

features of the isoflavones found in red clover which increases their binding affinity to aromatase thus inhibiting aromatase activity [13]. One of the isoflavones isolated from red clover, biochanin A, has been shown to have aromatase inhibitor activity at the transcriptional level [14]. Therefore, phytoestrogens may act as oestrogen antagonists through various mechanisms and therefore have an opposite effect on insulin sensitivity compared to oestrogen therapy.

One of the potential limitations of this study is that the effects of the currently studied red clover based phytoestrogen (Promensil), on insulin sensitivity, may be different from the effects of soy based oral phytoestrogens. For this study, a red clover based phytoestrogen was chosen because it is one of the most widely distributed phytoestrogens currently sold as an alternative to hormone replacement therapy for postmenopausal women. Red clover phytoestrogens are composed of the isoflavones present in soy, genistein and daidzein, as well as the soy isoflavone precursors, biochanin $\mathrm{A}$ and formomonectin. One small randomized, placebocontrolled study showed that the absorption of isoflavones from soy was similar to that of red clover [15].

In summary, after 12 weeks of drug therapy, oral phytoestrogen decreased insulin sensitivity in healthy nondiabetic postmenopausal women. Although red clover phytoestrogens may have a total isoflavone content similar to soy based phytoestrogens, this may not necessarily apply to other forms of phytoestrogens. The clinical significance of this finding highlights possible adverse side effects of alternatives to oestrogen therapy that warrant further evaluation in future studies.

\section{Acknowledgements}

We thank Kathy Jarvenpa, RN and the staff of the University of Michigan General Clinical Research Center for their care of 
our subjects during this study; Marla Smith for her technical assistance; and the women who took time to participate in this research study. This work was supported in part by a Merck/American Federation for Aging Research Junior Investigator Award in Geriatric Clinical Pharmacology, by a VA Research Career Development Award, by the General Clinical Research Center (NIH grant RR-00042), by the Michigan Diabetes Research and Training Center (NIDDK P60DK2572), by the Department of Veterans Affairs Ann Arbor and Greater Los Angeles Geriatric Research Education and Clinical Centers, and by the Claude D. Pepper Older Americans Center at the University of Michigan (AG 08808).

\section{Conflict of Interest}

None of the authors report any conflict of interest. C. C. L. did the design. Data collection and conduct was by C. C. L., C. J. B., and J. Z. K-V. C. C. L., J. Z. K-V., and L-J. L. did the analysis. Writing of manuscript was by C. C. L., C. J. B., J. Z. K-V., and L-J. L.

\section{References}

1. Booth NL, Piersen CE, Banuvar S, Geller SE, Shulman LP, Farnsworth NR. Clinical studies of red clover (Trifolium pratense) dietary supplements in menopause: a literature review. Menopause 2006; 13: 251-264.

2. Boonkasemsanti W, Reinprayoon D, Pruksananonda K et al. The effect of transdermal oestradiol on bleeding pattern, hormonal profiles and sex steroid receptor distribution in the endometrium of Norplant users. Hum Reprod 1996; 11(Suppl. 2): 115-123.

3. Blakesmith SJ, Lyons-Wall PM, George C, Joannou GE, Petocz P, Samman S. Effects of supplementation with purified red clover (Trifolium pratense) isoflavones on plasma lipids and insulin resistance in healthy premenopausal women. Br J Nutr 2003; 89: 467-474.

4. Anderson JW, Johnstone BM, Cook-Newell ME. Meta-analysis of the effects of soy protein intake on serum lipids. N Engl J Med 1995; 333: 276-282.
5. Cagnacci A, Soldani R, Carriero PL, Paoletti AM, Fioretti P, Melis GB. Effects of low doses of transdermal 17 beta-estradiol on carbohydrate metabolism in postmenopausal women. J Clin Endocrinol Metab 1992; 74 : 1396-1400.

6. Lindheim SR, Presser SC, Ditkoff EC, Vijod MA, Stanczyk FZ, Lobo RA. A possible bimodal effect of estrogen on insulin sensitivity in postmenopausal women and the attenuating effect of added progestin. Fertil Steril 1993; 60: 664-667.

7. Katz A, Nambi SS, Mather $K$ et al. Quantitative insulin sensitivity check index: a simple, accurate method for assessing insulin sensitivity in humans. J Clin Endocrinol Metabol 2000; 85: 2402-2410.

8. Jayagopal V, Albertazzi P, Kilpatrick ES et al. Beneficial effects of soy phytoestrogen intake in postmenopausal women with type 2 diabetes. Diabetes Care 2002; 25: 1709-1714.

9. Crisafulli A, Altavilla D, Marini H et al. Effects of the phytoestrogen genistein on cardiovascular risk factors in postmenopausal women. Menopause 2005; 12: 186-192

10. Lee CC, Kasa-Vubu JZ, Supiano MA. Differential effects of raloxifene and estrogen on insulin sensitivity in postmenopausal women. J Am Geriatr Soc 2003; 51: 683-688.

11. Adlercreutz H, Hockerstedt K, Bannwart C et al. Effect of dietary components, including lignans and phytoestrogens, on enterohepatic circulation and liver metabolism of estrogens and on sex hormone binding globulin (SHBG). J Steroid Biochem 1987; 27: 1135-1144.

12. Turner JV, Agatonovic-Kustrin S, Glass BD. Molecular aspects of phytoestrogen selective binding at estrogen receptors. J Pharm Sci 2007; 96: 1879-1885

13. Kao YC, Zhou C, Sherman M, Laughton CA, Chen S. Molecular basis of the inhibition of human aromatase (estrogen synthetase) by flavone and isoflavone phytoestrogens: a site-directed mutagenesis study. Environ Health Perspect 1998; 106: 85-92.

14. Wang Y, Man Gho W, Chan FL, Chen S, Leung LK. The red clover (Trifolium pratense) isoflavone biochanin $\mathrm{A}$ inhibits aromatase activity and expression. Br J Nutr 2008; 99: 303-310.

15. Tsunoda N, Pomeroy S, Nestel P. Absorption in humans of isoflavones from soy and red clover is similar. J Nutr 2002; 132: 2199-2201. 